\title{
Breathing retraining with chest wall mobilization improves respiratory reserve and decreases hyperactivity of accessory breathing muscles during respiratory excursions: A randomized controlled trial
}

\author{
MONTICHA SAKUnA, KeERIN MEKHORA*, WATtANA JALAJONDEJA, ChUTima JALAJONDEJA \\ Faculty of Physical Therapy, Mahidol University, Nakhon Pathom, Thailand.
}

\begin{abstract}
Purpose: The aim of this paper was to evaluate the effects of breathing retraining with chest wall mobilization on the onset of accessory breathing muscle recruitment and respiratory reserve in individuals with chronic neck pain. Methods: Thirty-two participants with non-specific chronic neck pain were randomly assigned into intervention and control groups. The intervention group received 30 minutes of breathing retraining with chest wall mobilization and the control group was assigned to rest for 30 minutes. Electromyography (EMG) of upper trapezius (UT), scalene (SC), and sternocleidomastoid (SCM) muscles were recorded during respiratory excursions by cycling for 12 minutes. Measurement of maximum voluntary ventilation (MVV), chest expansion, and pain intensity were taken during normal breathing. The immediate effects within each group and between two groups were analyzed. Results: Significant improvement in respiratory reserve was observed in the intervention group compared to control group through prolonged EMG onset of accessory breathing muscles. Moreover, increase of MVV, chest expansion and decrease in pain intensity were observed. Conclusions: This research suggests that breathing patterns and chest expansion should be considered within the physical assessment of breathing retraining, and that chest wall mobilization offers clinically important improvements in patients with chronic neck pain.
\end{abstract}

Key words: breathing retraining, neck pain, breathing re-education, respiratory reserve, breathing dysfunction

\section{Introduction}

Neck pain is one of the most common problems found in the general population [3]. Prevalence of neck pain has been reported to range between 0.4 to $86.8 \%$ [12], with between 70 and $80 \%$ of the population experiencing neck pain at least once in a lifetime [4], [10]. Neck pain impacts on not only an individuals' quality of life, but also on families, society, business, and health care systems. Several studies have demonstrated various manifestations of dysfunction associated with chronic neck pain such as alteration of neck muscle activity [9], increased forward head posture, decreased respiratory muscles strength [15], and changes in rib cage mechanics through the reduction of cervi- cal and thoracic spine stability [15]. In addition, many recent studies have also reported the decrease of maximum voluntary ventilation (MVV) [5], [21], [22]. Recently, further evidence of breathing dysfunction in patients with chronic neck pain has been reported and its clinical importance has been emphasized [5]-[8], [15], [16], [18], [21], [22]. The patients with chronic neck pain demonstrate the inhibition of deep cervical flexor muscles accompanied by an increased activation of superficial neck muscles, such as scalene (SC), sternocleidomastoid (SCM), and upper trapezius (UT) [9], [14]. Interestingly, these superficial neck muscles also play a role as accessory breathing muscles and their increased activity may lead to the alteration of breathing patterns and subsequent dysfunction, which have been reported in patients with chronic neck pain

\footnotetext{
* Corresponding author: Keerin Mekhora, Faculty of Physical Therapy, Mahidol University, 999 Phuttamonthon 4 Road, Salaya, Nakhon Pathom, 73170, Thailand. Phone: (66) 441-5450 ext 21603, fax: (66)2441-5454, e-mail: keerin.mekh@gmail.com

Received: May 2nd, 2020

Accepted for publication: July 9th, 2020
} 
[9]. Moreover, it has been reported that a decrease in thoracic mobility, chest expansion, and MVV, which provides evidence that underlines the relationship between breathing dysfunction and chronic neck pain [21], [22].

Impaired neck proprioception in patients with neck pain has been reported to cause segmental instability of the cervical and thoracic spine [2]. As there is a link between cervical and thoracic spine motion, muscles around the cervical spine, particularly accessory breathing muscles such as SC, SCM that are attached in this area, an alteration of neck muscle function has been proposed to be one of the causes of the altered movement patterns of the thoracic spine and rib cage [19]. The optimal breathing pattern allows for maximal lung expansion and capacity [1] that might decrease activity of accessory breathing muscles, facilitate deep cervical flexor muscles, and improve cervical segmental stability.

Previous study showed that breathing retraining can improve neck muscle activity, movement patterns, and reductions in pain intensity in chronic neck pain [22]. However, the study was observed during normal breathing and the intervention considered breathing retraining, but did not include chest wall mobilization. Thus, the aim of this study was to evaluate the immediate effect of breathing retraining with chest wall mobilization on neck muscle activities during respiratory excursions. Additionally, related parameters, such as mechanical respiratory function and pain, were investigated. It was hypothesized that maximizing the capacity of respiration by breathing retraining with chest wall mobilization might reduce hyperactivity of accessory breathing muscles by prolonging the onset of these muscles during heavy respiration.

\section{Materials and methods}

\subsection{Subjects}

Participants of this study were recruited from the Physical Therapy Clinic, Mahidol University. The protocol was approved by the Institutional Review Board (IRB), Mahidol University. A single assessor was responsible for screening for eligibility, taking consent and recording the outcome measures including EMG, MVV, chest expansion, and pain. Participant inclusion criteria were: at least a 6 month history of neck pain, mild to moderate pain intensity (VAS scale 2-7), body mass index (BMI) $18.5-24.9 \mathrm{~kg} / \mathrm{m}^{2}$, and age between 18 and 45 years old. The exclusion criteria were: severe pain (VAS scale $>7$ ), any neurological symptoms or known contraindications. All participants read the information sheet and signed a consent form before participation started.

\subsection{Experimental design}

The design of this study was a randomized controlled trial with single blinded outcome assessments, Thai Clinical Trial registry (TCTR) identification number (TCTR20180919005).

\subsection{EMG data collection procedure and processing}

Participants were asked to sit on a chair and perform deep breathing though a triflow for five repetitions. The maximum repetition of recorded EMG activities was selected for data processing. The participants were then asked to lie down in a supine position. The neck muscle activity was recorded for one minute during normal breathing. Muscle activity was then recorded for approximately 12 minutes during a submaximal cycling protocol on a stationary cycle ergometer (Monark 828E).

Myoelectric signals were acquired from the SCM, anterior SC, and UT muscles bilaterally using dual self-adhesive Ag-Agcl snap electrodes with an interelectrode spacing of $2 \mathrm{~cm}$ (The Telemyo Direct Transmition System, Naroxon INC, USA (C) 2012). Signals were amplified by $1000 \mathrm{~Hz}$, passed through a $10-500 \mathrm{~Hz}$ bandwidth filter [20], sampled at $1500 \mathrm{~Hz}$, and an inter-electrode impedance below $2 \mathrm{k} \Omega$ [13]. The EMG data were full wave rectified and filtered using a root mean square (RMS) with a window length of $100 \mathrm{~ms}$ to produce an enveloped EMG signal. The EMG onset of each muscle was determined by a signal greater than 3 standard deviations of the baseline values and when the duration of the contraction was greater than 25 milliseconds [11]. The average EMG amplitude was collected from the muscle activation after EMG onset untill the end of test. The resulting data were then normalized to the percentage of MVC during deep breathing using the triflow.

\subsection{MVV data collection procedure}

The MVV was measured by spirometer (Spirolab III, MIR srl Medical International Research Inc., Roma 
Italy) according to The American Thoracic Society (ATS) guidelines. All participants were familiarized with the test before starting the procedure. The standard protocol of MVV test was applied to all participants. Participants performed at least three trials with one minute of rest between each trial. Verbal encouragement was applied to all participants to achieve a maximum effort. The best test value was used for data analysis.

\subsection{Chest expansion data collection procedure}

Chest expansion was measured at the level of the xiphoid process and 10th costal cartilage with a flat measuring tape. The difference of circumference between maximal inspiration and maximal expiration was measured twice. The mean of the two values was recorded. If the difference between the two trials was greater than $1 \mathrm{~cm}$, a $3 \mathrm{rd}$ measurement was taken, and the average of the 2 largest values was recorded. The intraclass correlation coefficients (ICC) value of chest expansion of 0.77 indicated good reliability.

\subsection{Pain intensity data collection procedure}

Pain intensity was rated by using a visual analog scale (VAS). This scale is a self-report instrument that consists of a 100-mm straight line rating from no pain on the left side to worst possible pain on the right side.

A therapist with a post-graduate qualification in manual musculoskeletal therapy was responsible for randomly assigning participants into an intervention and control group, and provided the breathing retraining and chest wall mobilization as a treatment. Before treatment, the therapist performed a subjective and objective physical examination, observing the participants' respiration in both sitting and lying positions, looking for abnormal breathing patterns in the upper, lower or lateral movement of the chest wall. The therapist then asked all participants to perform a breathing retraining protocol with chest wall mobilization in the different directions depending on the finding from the examination. If participant had more pain, breathing retraining was provided first. However, if the participant had more chest wall stiffness, chest wall mobilization was performed first. However, in all cases, the treatment took no longer than 30 minutes.

\subsection{Data analysis}

Data were analyzed with the Statistical Package for the Social Sciences (SPSS) version 18 for Windows. Descriptive statistics were used to summarize all outcome measures. The distribution of the quantitative data was assessed using the Kolmogorov-Smirnov test. When the data were found suitable for parametric testing, independent $t$-tests were used to explore differences between groups and paired $t$-tests were used to determine changes between pre and post. If the data were found to be not normally distributed, Mann-Whitney $U$-tests and Wilcoxon signed ranks tests were used, respectively. The significance level for all outcomes was set at 0.05 .

\section{Results}

Participants with chronic neck pain (7 males and 25 females) were randomly assigned into an intervention and control group. Each group contained with 16 volunteers. The characteristics of the participants were shown in Table 1.

Table 1. Demographic data of the participants at baseline

\begin{tabular}{|c|c|c|c|}
\hline & $\begin{array}{c}\text { Intervention } \\
\quad(n=16)\end{array}$ & $\begin{array}{l}\text { Control } \\
(n=16)\end{array}$ & $p$-value \\
\hline \multicolumn{4}{|l|}{ Gender } \\
\hline Male & 3 & 4 & \\
\hline Female & 13 & 12 & \\
\hline Age [years] & $23.90(4.6)$ & $27.38(6.7)$ & $0.26_{\mathrm{b}}$ \\
\hline Weight $[\mathrm{kg}]$ & $57.40(9.5)$ & $58.80(8.5)$ & $0.68_{\mathrm{a}}$ \\
\hline High $[\mathrm{cm}]$ & $162.50(7.9)$ & $163.20(6.9)$ & $0.78_{\mathrm{a}}$ \\
\hline BMI & $21.64(2.1)$ & $21.90(2.1)$ & $0.67_{\mathrm{a}}$ \\
\hline \multicolumn{4}{|c|}{ Average EMG amplitude during normal breathing (\%) } \\
\hline Rt. UT m & $20.69(12.87)$ & $18.80(13.67)$ & $0.69_{\mathrm{b}}$ \\
\hline Rt. SC m & $10.35(16.37)$ & $6.38(6.58)$ & $0.45_{\mathrm{a}}$ \\
\hline Rt. SCM m & $16.36(18.56)$ & $10.65(16.14)$ & $0.14_{a}$ \\
\hline Lt. UT m & $20.50(12.01)$ & $28.65(29.72)$ & $0.62_{\mathrm{a}}$ \\
\hline Lt. SC m & $10.20(7.76)$ & $12.17(18.79)$ & $0.26_{\mathrm{a}}$ \\
\hline Lt. SCM m & $11.54(6.26)$ & $12.92(22.80)$ & $0.82_{\mathrm{b}}$ \\
\hline \multicolumn{4}{|l|}{ Pain VAS $(0-100 \mathrm{~mm})$} \\
\hline At rest & $2.30(2.1)$ & $3.00(1)$ & $0.37_{\mathrm{a}}$ \\
\hline On worst movement & $4.70(2.4)$ & $4.80(2.1)$ & $0.89_{\mathrm{b}}$ \\
\hline
\end{tabular}

a - Statistical analysis were performed using Mann-Whitney $U$-test, $\mathrm{b}$ - Statistical analysis were performed using the independent $t$-test, significant $*-p<0.05, \mathrm{~m}-$ muscle, Rt. - Right, Lt. - Left, UT - Upper Trapezius, SC - Scalene, SCM - Sternocleidomastoid. 
No significant differences were found in average age, weight, high, body mass index (BMI), average electromyography (EMG) amplitude during normal breathing, and pain at baseline between the two groups.

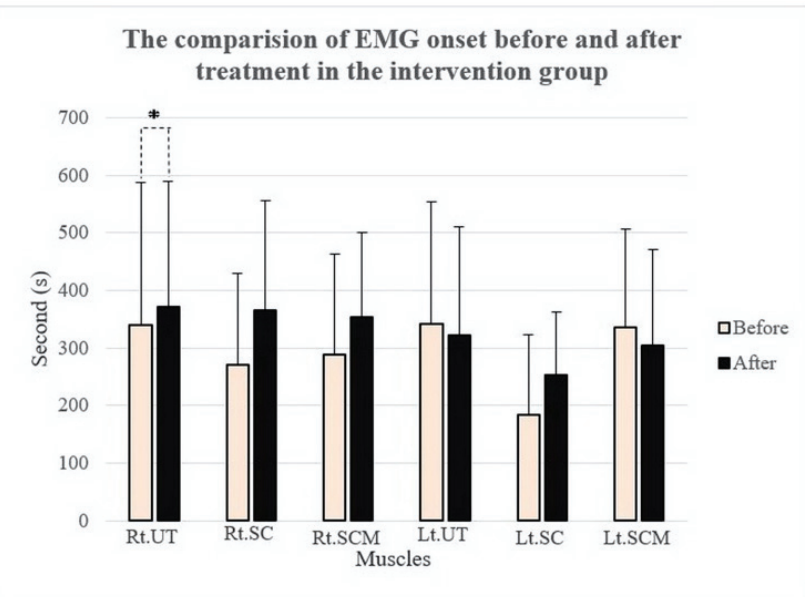

Fig. 1. Comparison of EMG onset before and after treatment in the intervention group $(*=p<0.05)$

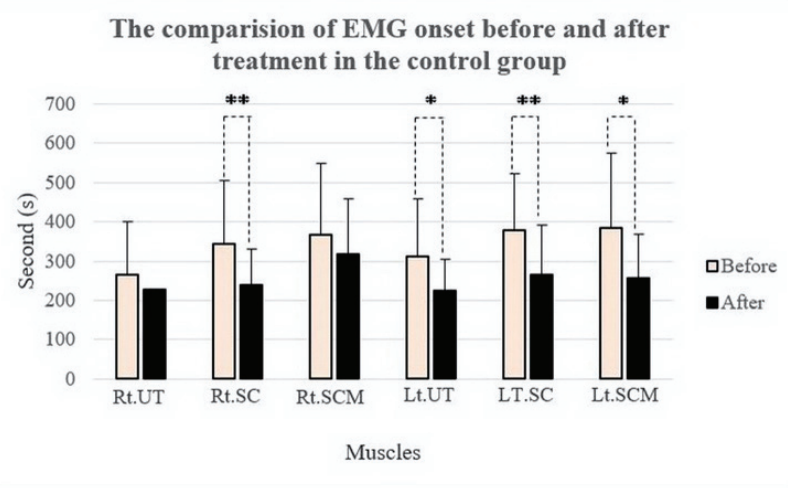

Fig. 2. Comparison of EMG onset before and after treatment in the control group $(*=p<0.05, * *=p<0.01)$

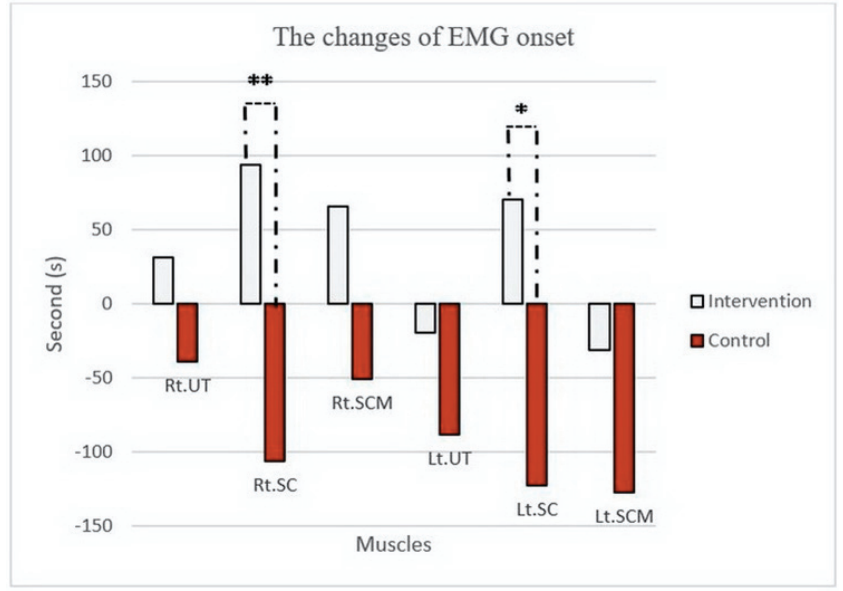

Fig. 3. The changes of EMG onset compared between intervention and control groups $(*=p<0.05, * *=p<0.01)$

The results demonstrated significant prolonged EMG onset of right UT and found their trend towards almost of accessory breathing muscles (right UT, right $\mathrm{SC}$, right SCM, and left SC), except for left UT and left SCM muscles (Fig. 1). The shortened EMG onset were found in all muscles in the control group (Fig. 2). The significant differences of EMG onset of SC muscles between two groups were seen, however, there was no statistical significant differences of UT and SCM muscles between two groups (Fig. 3).

This study found a significant increase in the MVV in the intervention group (91.14 to $94.96 \mathrm{l} / \mathrm{min}, p=$ 0.041) (Table 2). On the contrary, a significant decrease in chest expansion were found in the control group. However, this study showed no statistical significant differences of MVV between two groups (Table 3).

There was no statistically significant difference between pre- and post-tests in chest expansion for the

Table 2. Comparison of MVV, chest expansion and pain between before and after treatment in each group

\begin{tabular}{|l|c|c|c|c|c|c|}
\hline \multicolumn{1}{|l|}{ Outcome measure } & \multicolumn{3}{|c|}{ Intervention $(n=16)$} & \multicolumn{3}{c|}{ Control $(n=16)$} \\
\cline { 1 - 6 } [mean (SD)] & before & after & $p$-value & before & after & $p$-value \\
\hline MVV [L/min] & $\begin{array}{c}91.14 \\
(19.68)\end{array}$ & $\begin{array}{c}94.96 \\
(20.95)\end{array}$ & $0.041^{\mathrm{b} *}$ & $\begin{array}{c}99.05 \\
(17.53)\end{array}$ & $\begin{array}{c}99.26 \\
(14.58)\end{array}$ & $0.865^{\mathrm{b}}$ \\
\hline Chest expansion [cm] & $\begin{array}{c}0.95 \\
(0.69)\end{array}$ & $\begin{array}{c}1.04 \\
(0.65)\end{array}$ & $0.207^{\mathrm{b}}$ & $\begin{array}{c}0.92 \\
(0.53)\end{array}$ & $\begin{array}{c}0.77 \\
(0.45)\end{array}$ & $0.011^{\mathrm{b} *}$ \\
\hline Pain at rest & $\begin{array}{c}2.3 \\
(2.1)\end{array}$ & $\begin{array}{c}0.8 \\
(1)\end{array}$ & $0.001^{\mathrm{a} * *}$ & $\begin{array}{c}3.0 \\
(1.0)\end{array}$ & $\begin{array}{c}2.8 \\
(2.6)\end{array}$ & $0.768^{\mathrm{b}}$ \\
\hline Pain on worst movement & $\begin{array}{c}4.7 \\
(2.4)\end{array}$ & $\begin{array}{c}2.1 \\
(2.5)\end{array}$ & $0.000^{\mathrm{a} * *}$ & $\begin{array}{c}4.8 \\
(2.1)\end{array}$ & $\begin{array}{c}3.8 \\
(2.8)\end{array}$ & $0.062^{\mathrm{a}}$ \\
\hline
\end{tabular}

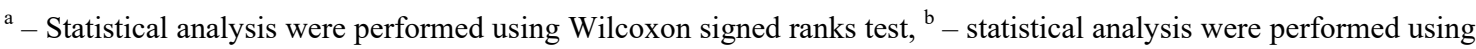
the paired $t$-test, significant $*-p<0.05$, significant $* *-p<0.01$. 
intervention group. However, the significant decrease of chest expansion in the control group was found (Table 2). The significant differences of chest expansion was found $(p=0.009)$ between the two groups (Table 3).

Table 3. Comparisons of the changes of MVV, chest expansion and pain between two groups

\begin{tabular}{|l|c|c|c|}
\hline Outcome measure & \multicolumn{3}{|c|}{ Difference scores } \\
\hline [mean (SD)] & $\begin{array}{c}\text { intervention } \\
(n=16)\end{array}$ & $\begin{array}{c}\text { control } \\
(n=16)\end{array}$ & $p$-value \\
\hline MVV [L/min] & $3.82(6.82)$ & $0.21(4.76)$ & $0.093^{\mathrm{b}}$ \\
\hline Chest expansion [cm] & $0.09(0.27)$ & $-0.15(0.21)$ & $0.009^{\mathrm{b}} * *$ \\
\hline Pain at rest & $-1.5(1.2)$ & $-0.2(2.2)$ & $0.048^{\mathrm{b} *}$ \\
\hline Pain on worst movement & $-2.7(1.1)$ & $-0.9(2.5)$ & $0.002^{\mathrm{a} * *}$ \\
\hline
\end{tabular}

a - statistical analysis were performed using Mann-Whitney $U$-test, ${ }^{\mathrm{b}}$ - statistical analysis were performed using the independent $t$-test, significant $*-p<0.05$, significant $* *-p<0.01$.

A significant reduction in pain at rest and pain on worst movement were only seen in the intervention group ( $p=0.001$ and 0.000 , respectively), but no significant differences were seen in control group (Table 2). The results demonstrated the significant differences of pain at rest and pain on worst movement ( $p=0.048$ and $p=0.002$, respectively) between two groups (Table 3).

\section{Discussion}

This study aimed to evaluate the effect of breathing retraining using chest wall mobilization on the recruitment of accessory breathing muscles and respiratory reserve during respiratory excursions. According to the findings, participants with chronic neck pain who received breathing retraining with chest wall mobilization had a significantly increased capacity of mechanical respiratory function during high respiratory load, increased MVV, increased chest expansion, and reduced pain intensity.

Several studies have shown that patients with chronic neck pain present the association between breathing dysfunction and many motor function alterations [8], [15], [21]. However, few studies have investigated the consequence of using breathing retraining as a treatment in patients with chronic neck pain [22]. To the best of our knowledge, there has been no evidence of effects of breathing retraining on accessory breathing muscle during high respira- tory load. The accessory breathing muscles have to play a role when the body need respiratory reserve to meet the physiologic demands of body oxygen requirements, and patients with chronic neck pain demonstrate an increased activation of accessory breathing muscles [9], [14] and might show on early onset of muscle activity. Correct mechanical respiratory function, including the work of the diaphragm and flexibility of rib and chest expansion, might reduce accessory breathing muscle activity or prolong their onset. A trend was demonstrated towards a delayed onset in most accessory breathing muscles in the intervention group, indicating an improvement in the mechanical respiratory function. However, it was surprising that more than half of the muscles tested in the control group also revealed statistically significant earlier EMG onset, indicating higher muscle activity in response to the respiratory load. This could possibly be explained by some residual effect after the cycling task, such as tiredness or exhaustion, which could have influenced the performance of the respiration, however, this effect was only seen in the control group.

The previous study reported that changing the position of head decreases respiratory movement of the lower chest and increases movement of upper chest [19]. The preliminary findings by Yeampattanaporn et al. [22] showed an increase of the lower chest expansion and decrease of pain intensity after breathing retraining in participants with chronic neck pain. The results of this current study can be compared indirectly with Yeampattanaporn et al., although there were some methodological and treatment differences. These included not using high respiratory load or chest mobilization, however the results of this current study support their conclusions. The intervention group revealed no significant difference of chest expansion, however, slightly higher scores was found in post-test. On the contrary, the control group showed a significant decrease in chest expansion. It could be explained by the increase response of respiration to some residual effect after the cycling task, leading to the accessory breathing muscles recruitment, with increased sternocleidomastoid muscle activity, causing rib cage elevation, reducing thoracoabdominal mobility, and the upper chest was used more than lower chest, as described in breathing dysfunction pattern [18]. The chest expansion were measured near the 10th rib, which reflected the lower chest movement. Therefore, the reduction of chest expansion found in the control group and the trend of increase chest expansion found in the intervention group in this study implied for the benefit of the treatment that improves the biomechan- 
ics of breathing. However, this study had the limitation of detecting the changes of upper and middle chest movement.

MVV was significantly correlated with chest expansion and chest wall mobility was the significant predictor of MVV [21]. However, the previous study [22] showed no significant increase in MVV, as opposed to this study. Because of this study included chest wall mobilization to the treatment, result in cumulative effects on lung compliance and mobility, hence, the increase of MVV, was seen.

The limitation of this study was the treatment time that was set at only 30 minutes. Some patients need more time for producing the improvement. The long-term study was suggested for the next future research to see more effects of the treatment. Moreover, there was some residual effect after the cycling task. If participants have more time to rest, the shortened EMG onset may be not seen in the control group and prolonged EMG onset may be obviously seen in the intervention group. Another limitation was the measurement method for chest expansion that was measured only at the lower chest, so it cannot isolate the changes of upper and middle chest. The future study may try to measure the isolated changes of upper, middle, and lower chest by another method. Moreover, the therapist observed participants' respiration only to consider the treatment technique tailored to individual. We recommended for the future study to categorize the type or the severity of breathing dysfunction before randomizing each participant to both groups. It might pronounce more effects of the treatment.

\section{Conclusions}

This study suggested that breathing pattern and the chest expansion should be concerned as one of neck pain assessment. Breathing retraining with chest wall mobilization encourage good respiratory work, reduce hyperactivity of accessory breathing muscles and then reduce pain in chronic neck pain.

\section{Acknowledgements}

The authors thank the Faculty of Physical Therapy, Mahidol University (Thailand) for their kind support, and all participants for their involvement in this research.

\section{References}

[1] Chaitow L., Breathing pattern disorders, motor control, and low back pain, J. Osteopath. Med., 2004, 7 (1), 33-40, DOI: 10.1016/S1443-8461(04)80007-8.

[2] Comerford M.J., MotTram S.L., Movement and stability dysfunction - contemporary developments, Man. Ther., 2001, 6 (1), 15-26, DOI: 10.1054/math.2000.0388.

[3] CотE P. et al., The annual incidence and cause of neck pain in the general population: a population-based cohort study, Pain, 2004, 112(3), 267-273, DOI: 10.1016/j.pain.2004.09.004.

[4] Cote P., CASSidy J.D., CARRoll L., The epidemiology of neck pain: what we have learned from our population-based studies, J. Can. Chiropr. Assoc., 2003, 47 (4), 284-290.

[5] COURTNEY R., The functions of breathing and its dysfunctions and their relationship to breathing therapy, Int. J. Osteopath. Med., 2009, 12 (3), 78-85, DOI: 10.1016/ j.ijosm.2009.04.002.

[6] Dimitriadis Z., Hypocapnia in patients with chronic neck pain: association with pain, muscle function, and psychologic states, Am. J. Phys. Med. Rehabil., 2013. 92 (9), 746-754, DOI: 10.1097/PHM.0b013e31829e74f7.

[7] Dimitriadis Z., Pulmonary function of patients with chronic neck pain: a spirometry study, Respir. Care, 2014, 59 (4), 543-549, DOI: 10.4187/respcare.01828.

[8] DimitRIADIS Z., Respiratory weakness in patients with chronic neck pain, Man. Ther., 2012, 18 (3), 248-253, DOI: 10.1016/j.math.2012.10.014.

[9] Falla D., BilenkiJ G., Jull G., Patients with chronic neck pain demonstrate altered patterns of muscle activation during performance of a functional upper limb task, Spine, 2004, 29 (13), 1436-1440, DOI: 10.1097/01.brs.0000128759.02487.bf.

[10] FERRARI R., RUSSELl A.S., Regional musculoskeletal conditions: neck pain, Best Pract. Res. Clin. Rheumatol., 2003, 17 (1), 57-70, DOI: 10.1016/s1521-6942(02)00097-9.

[11] Hodges P.W., Bui B.H., A comparison of computer-based methods for the determination of onset of muscle contraction using electromyography, Electroencephalogr. Clin. Neurophysiol., 1996, 101 (6), 511-519, DOI: 10.1016/ s0013-4694(96)95190-5.

[12] Hoy D.G., The epidemiology of neck pain, Best Pract. Res. Clin. Rheumatol., 2010, 2 (6), 783-792, DOI: 10.1016/ j.berh.2011.01.019.

[13] Hug F., Optimized analysis of surface electromyograms of the scalenes during quiet breathing in humans, Resp. Physiol. Neurobi., 2006, 150(1), 75-81, DOI: 10.1016/ j.resp.2005.04.008.

[14] Jull G., Falla D., Does increased superficial neck flexor activity in the craniocervical flexion test reflect reduced deep flexor activity in people with neck pain?, Man. Ther., 2016, 25, 43-47, DOI: 10.1016/j.math.2016.05.336.

[15] KAPRELI E., Respiratory dysfunction in chronic neck pain patients. A pilot study, Cephalalgia, 2009, 29 (7), 701-710, DOI: $10.1111 /$ j.1468-2982.2008.01787.x.

[16] Kapreli E., Vourazanis E., Strimpakos N., Neck pain causes respiratory dysfunction, Med. Hypotheses, 2008, 70 (5), 1009-1013, DOI: 10.1016/j.mehy.2007.07.050.

[17] MCLaughlin L., Breathing evaluation and retraining in manual therapy, J. Bodyw. Mov. Ther., 2009, 13 (3), 276-282, DOI: 10.1016/j.jbmt.2009.01.005. 
[18] Perri M.A., Halford E., Pain and faulty breathing: a pilot study, J. Bodyw. Mov. Ther., 2004. 8 (4), 297-306, DOI: 10.1016/S1360-8592(03)00085-8.

[19] SzCZYGiEŁ E. et al., Biomechanical influences on head posture and the respiratory movements of the chest, Acta Bioeng. Biomech., 2015, 17 (2), 143-148, DOI: 10.5277/ ABB-00118-2014-02.

[20] Tsang S.M.H., Szeto G.P.Y., LeE R.Y.W., Altered spinal kinematics and muscle recruitment pattern of the cervical and thoracic spine in people with chronic neck pain during functional task, J. Electromyogr. Kinesiol., 2014, 24 (1), 104-113, DOI: 10.1016/j.jelekin.2013.10.011.

[21] WIRTH B., Respiratory dysfunction in patients with chronic neck pain - Influence of thoracic spine and chest mobility, Man. Ther., 2014, 9(15), 440-444, DOI: 10.1016/j.math.2014.04.011.

[22] YeAmPATTANAPORN O., Immediate effects of breathing re-education on respiratory function and range of motion in chronic neck pain, J. Med. Assoc. Thai., 2014, 97, Suppl 7, S55-9. 